Research Paper

\title{
Adaptation to noise in amplitude modulation detection without the medial olivocochlear reflex
}

\author{
Miriam I. Marrufo-Pérez a, b , Almudena Eustaquio-Martín ${ }^{\text {a, b }}$, Milagros J. Fumero a, b, \\ José M. Gorospe ${ }^{\mathrm{a}, \mathrm{b}, \mathrm{c}, \mathrm{d}}$, Rubén Polo ${ }^{\mathrm{e}}$, Auxiliadora Gutiérrez Revilla ${ }^{\mathrm{e}}$, \\ Enrique A. Lopez-Poveda a, b, c, * \\ a Instituto de Neurociencias de Castilla y León, Universidad de Salamanca, Salamanca, Spain \\ b Instituto de Investigación Biomédica de Salamanca, Universidad de Salamanca, Salamanca, Spain \\ c Departamento de Cirugía, Universidad de Salamanca, Salamanca, Spain \\ d Servicio de Otorrinolaringología, Complejo Asistencial Universitario de Salamanca, Salamanca, Spain \\ e Servicio de Otorrinolaringología, Hospital Universitario Ramón y Cajal, Madrid, Spain
}

\section{A R T I C L E I N F O}

\section{Article history:}

Received 25 October 2018

Received in revised form 5 March 2019

Accepted 19 March 2019

Available online 22 March 2019

\section{Keywords:}

Cochlear implant

Temporal effect

Noise adaptation

Sound envelope

Dynamic range adaptation

Modulation detection interference

\begin{abstract}
A B S T R A C T
The detection of amplitude modulation (AM) in quiet or in noise improves when the AM carrier is preceded by noise, an effect that has been attributed to the medial olivocochlear reflex (MOCR). We investigate whether this improvement can occur without the MOCR by measuring AM sensitivity for cochlear implant $(\mathrm{CI})$ users, whose MOCR effects are circumvented as a result of the electrical stimulation provided by the CI. AM detection thresholds were measured monaurally for short $(50 \mathrm{~ms})$ AM probes presented at the onset (early condition) or delayed by $300 \mathrm{~ms}$ (late condition) from the onset of a broadband noise. The noise was presented ipsilaterally, contralaterally and bilaterally to the test ear. Stimuli were processed through an experimental, time-invariant sound processing strategy. On average, thresholds were $4 \mathrm{~dB}$ better in the late than in the early condition and the size of the improvement was similar for the three noise lateralities. The pattern and magnitude of the improvement was broadly consistent with that for normal hearing listeners [Marrufo-Pérez et al., 2018, J Assoc Res Otolaryngol 19:147-161]. Because the electrical stimulation provided by CIs is independent from the middle-ear muscle reflex (MEMR) or the MOCR, this shows that mechanisms other than the MEMR or the MOCR can facilitate AM detection in noisy backgrounds.
\end{abstract}

(c) 2019 Elsevier B.V. All rights reserved.

\section{Introduction}

Many auditory perception tasks are harder in noisy than in quiet backgrounds. For some tasks, however, performance in noise improves as the probe sound is delayed from the noise onset, an effect sometimes referred to as "noise adaptation" (Cervera and Ainsworth, 2005; Marrufo-Pérez et al., 2018a). For example, listeners with normal hearing $(\mathrm{NH})$ recognize more syllables or words in noisy backgrounds when the speech tokens are delayed from the noise onset than when they start at the same time as the noise (Ben-David et al., 2012, 2016; Cervera and Ainsworth, 2005; Cervera and Gonzalez-Alvarez, 2007; Marrufo-Pérez et al., 2018a).

\footnotetext{
* Corresponding author. Instituto de Neurociencias de Castilla y León, Universidad de Salamanca, Calle Pintor Fernando Gallego 1, 37007, Salamanca, Spain

E-mail address: ealopezpoveda@usal.es (E.A. Lopez-Poveda).
}

Similarly, the sensitivity of NH listeners to amplitude modulation (AM) in quiet or in noise improves when the AM carrier is preceded by a few hundred milliseconds of steady-state noise (Almishaal et al., 2017; Jennings et al., 2018; Marrufo-Pérez et al., 2018b; Sheft and Yost, 1990; Viemeister, 1979). Several physiological mechanisms may underlie these improvements, including mechanisms mediated by the medial olivocochlear reflex (MOCR). The present study reevaluates the neural mechanisms responsible for adaptation to noise in AM detection. To that end, we measured AM sensitivity for cochlear implant users, whose MOCR effects are circumvented by the direct electrical stimulation of their auditory nerve.

The mammalian auditory nervous system contains afferent as well as efferent fibers. Some efferent fibers terminate in the bodies of outer hair cells (OHCs) in the cochlea (Warr and Guinan, 1979), and may be activated reflexively by ipsilateral and/or contralateral 
sounds (reviewed by Lopez-Poveda, 2018; Guinan, 1996). Activation of the MOCR could facilitate the detection of AM in quiet when the AM carrier is preceded by noise (Almishaal et al., 2017; Jennings et al., 2018; Marrufo-Pérez et al., 2018b). The idea is that the preceding noise activates the ipsilateral and/or the contralateral MOCR. Activation of the MOCR linearizes the response of the basilar membrane (BM) to the AM carrier at the cochlear region tuned to the carrier frequency (Cooper and Guinan, 2003, 2006; Murugasu and Russell, 1996), thus enhancing the effective AM depth as represented at the output of the BM. For an AM carrier embedded in noise, the MOCR could also enhance the AM depth as represented in the auditory nerve by restoring the dynamic range of auditory nerve fibers to values observed in quiet (Guinan, 2006; Winslow and Sachs, 1988; see Fig. 1 in Marrufo-Pérez et al., 2018b).

Some aspects in the data from previous studies seem consistent with the MOCR explanation. For example, Almishaal et al. (2017) reported worse (larger) AM detection thresholds when a short AM carrier was presented in quiet without than with an ipsilateral notched noise precursor at $40 \mathrm{~dB}$ sound pressure level (SPL). The difference was greater at mid than at lower or higher AM carrier levels, consistent with the precursor activating the ipsilateral MOCR, linearizing the BM responses at moderate-to-high levels (where the BM response is more compressive), and facilitating AM detection. Jennings et al. (2018) reported that hearing-impaired (HI) listeners (1) had better AM detection thresholds than NH listeners when a short AM carrier with a moderate level (around $65 \mathrm{~dB}$ SPL) was presented in quiet, ${ }^{1}$ but (2) showed smaller improvements in thresholds than $\mathrm{NH}$ listeners did when a precursor was used. Those results are consistent with $\mathrm{HI}$ listeners having less compressive BM responses and a smaller change in BM compression as a result of MOCR activation. Marrufo-Pérez et al. (2018b) reported worse AM detection thresholds for a short AM carrier in simultaneous steady-state noise when the AM carrier started at the same time as the noise than when the carrier was delayed $300 \mathrm{~ms}$ from the noise onset. This improvement occurred for a monaural AM carrier presented in ipsilateral, contralateral, and bilateral broadband noise (BBN) as well as in contralateral low-band noise (LBN). For contralateral noise, the improvement was less for LBN than for BBN, which is consistent with the reported smaller reductions in otoacoustic emission (OAE) levels (i.e., less linearization of BM responses by MOCR activation) for contralateral LBN than BBN (Maison et al., 2000).

Some aspects in the results of those studies, however, are not consistent with the MOCR explanation. For example, neural dynamic range adaptation could equally account for the better AM detection thresholds in the presence than in the absence of a noise precursor (Almishaal et al., 2017; see below). In addition, MarrufoPérez et al. (2018b) showed that the magnitude of the temporal improvement was similar for bilateral, ipsilateral and contralateral BBN, which appears inconsistent with the greater reductions in OAE levels when bilateral rather than ipsilateral or contralateral BBN is used to elicit the MOCR (Berlin et al., 1995).

A difficulty in interpreting the results for $\mathrm{NH}$ listeners is that $\mathrm{NH}$ listeners almost certainly have mechanisms other than the MOCR that can contribute to the temporal improvements. For example, the discharge rate of neurons in the auditory nerve (Wen et al., 2009, 2012), the inferior colliculus (Dean et al., 2005, 2008), and

\footnotetext{
1 While we describe the experiments of Almishaal et al. (2017) and Jennings et al. (2018) as AM detection in quiet, their AM probes were actually presented simultaneously with a notched noise. Our description is nonetheless justified because this notched noise was used to minimize off-frequency listening rather than to mask the AM probe and its level was so low $(50 \mathrm{~dB} / \mathrm{Hz}$ below the carrier spectrum level) that it was unlikely to mask the AM probe.
}

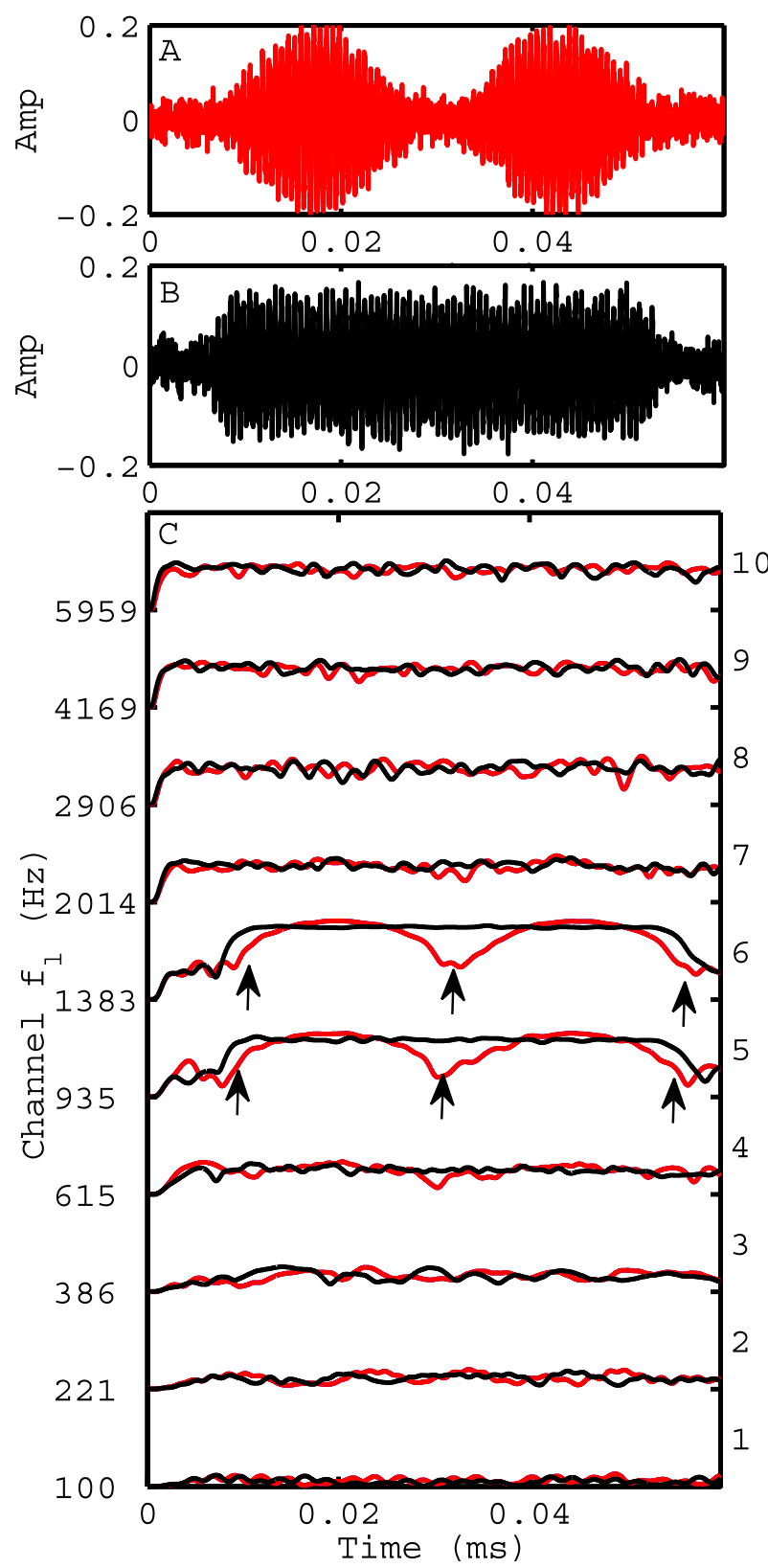

Fig. 1. Envelopes at the output of a 10-channel processor for an unmodulated and $100 \%$ modulated $(\mathrm{m}=1)$ carrier in ipsilateral noise. Carrier and noise levels were as in the experiments ( $-20 \mathrm{~dB}$ FS and $-30 \mathrm{~dB} F$, respectively). Unlike in the experiment, the carriers in this example were preceded and followed by $5 \mathrm{~ms}$ of noise. A. Modulated carrier. B. Unmodulated carrier. C. Output envelopes for each processor channel. Envelopes have been displaced vertically to avoid clutter. Arrows indicate the times and channels at which the modulated and unmodulated carriers produced the largest differences in amplitude. The numbers in the right and left ordinates indicate channel number and the lower 3-dB cutoff frequency of the channel bandpass filter, respectively.

the auditory cortex (Watkins and Barbour, 2008) adapt to the most frequently occurring level in the acoustic stimulus. This causes a horizontal shift of the neurons' rate-level functions toward the most frequent sound level, something that can facilitate the coding of AM and thus AM detection when the level of AM carrier is within the dynamic range of the adapted rate-level function (see Fig. $1 \mathrm{C}$ in Marrufo-Pérez et al., 2018b). The time course of dynamic range adaptation (100-400 ms) (Wen et al., 2012) is similar to the time course of MOCR activation $(277 \pm 62 \mathrm{~ms}$ ) (Backus and Guinan, 
2006). Hence, it is conceivable that mechanisms such as dynamic range adaptation can also contribute to the temporal improvements. Indeed, Marrufo-Pérez et al. (2018a) found temporal improvements in word-in-noise recognition that were similar for cochlear-implant $(\mathrm{CI})$ users and $\mathrm{NH}$ listeners tested with vocoded words. Because the electrical stimulation delivered by CIs is independent from the MOCR (Lopez-Poveda et al., 2016), their study shows that speech-in-noise recognition can improve over time without the MOCR. Furthermore, because only speech envelope cues were available to both $\mathrm{CI}$ users and $\mathrm{NH}$ listeners tested with vocoded words (temporal fine structure speech cues were absent), this suggests that AM detection might also improve over time without the MOCR.

The aim of the present study was to investigate this hypothesis. Here, we use the term "adaptation" to refer to any improvement in AM sensitivity in noise over time, regardless of the mechanism underlying this improvement. Our approach consisted of measuring the temporal improvement of AM-in-noise sensitivity for CI users. Because the MOCR exerts its inhibitory effect via the OHCs (Guinan, 1996), and the electrical stimulation delivered by CIs bypasses OHCs, MOCR effects are circumvented by the electrical stimulation delivered to CI users (Lopez-Poveda et al., 2016). Therefore, the existence of adaptation for CI users would show that AM-in-noise detection can improve over time without the MOCR. By contrast, if $\mathrm{CI}$ users do not show adaptation to noise, then this would suggest that the MOCR may play a role in adaptation to noise in AM detection. To make the comparison between $\mathrm{NH}$ and $\mathrm{CI}$ listeners as close as possible, we used an experimental design similar to that used by Marrufo-Pérez et al. (2018b) for NH listeners. In short, we compared AM detection thresholds for brief ( $50 \mathrm{~ms}$ ) carriers presented monaurally at the onset ('early' condition) and delayed $300 \mathrm{~ms}$ from the onset ('late' condition) of an ipsilateral, contralateral, and binaural (diotic) steady-state BBN.

\section{Methods}

\subsection{Participants}

Ten users (four women) of CIs manufactured by MED-EL GmbH (Austria) participated in the study. Eight of them wore bilateral CIs, and two of them wore a hearing aid in the ear opposite to the $\mathrm{CI}$ (Table 1). AM detection thresholds were measured monaurally in the implanted ear (unilateral CI users) or in the self-reported better ear (bilateral CI users).

Participants were volunteers and were not paid for their services. All of them signed an informed consent to participate in the study. Experimental procedures were approved by the Human Experimentation Ethics Committee of the University of Salamanca (Spain).

\subsection{Stimuli processing}

CI users were not tested with their clinical audio processors but with an experimental processing strategy that lacked automatic gain control or any other form of dynamic (time varying) processing that could have caused differences in AM thresholds between the early and late conditions (e.g., Lopez-Poveda et al., 2016). The strategy included a high-pass pre-emphasis filter (first-order Butterworth filter with a 3-dB cutoff frequency of $1.2 \mathrm{kHz}$ ); a bank of sixth-order Butterworth band-pass filters whose 3-dB cutoff frequencies followed a modified logarithmic distribution between 100 and $8500 \mathrm{~Hz}$; envelope extraction via full-wave rectification and low-pass filtering (fourth-order Butterworth low-pass filter with a 3-dB cutoff frequency of $400 \mathrm{~Hz}$ ); a fixed logarithmic compression function to map the wide dynamic range of sounds in the environment into the relatively narrow dynamic range of electrically evoked hearing (Boyd, 2006); and continuous interleaved sampling of compressed envelopes with biphasic electrical pulses (Wilson et al., 1991). The number of filters in the bank was identical to the number of active electrodes in the implant (Table 1), and equal between the left- and right-ear processors.

Before testing commenced, electrical current levels at maximum comfortable loudness (MCL) were measured using the method of adjustments. Minimum stimulation levels (i.e., thresholds) were set to 0,5 , or 10 percent of MCL values, according to each participant's preference (Boyd, 2006).

\subsection{Stimuli}

The mathematical expression describing the AM probe was:

$x(t)=\left[1+m \cdot \sin \left(2 \pi f_{\mathrm{m}} t+\phi\right)\right] \cdot \sin \left(2 \pi f_{\mathrm{c}} t\right)$

where $m$ is the modulation depth $(0 \leq m \leq 1), f_{m}$ is the modulation rate $(40 \mathrm{~Hz}), \phi$ is the starting phase of the modulation $(-\pi / 2), f_{c}$ is the carrier frequency $(1.5 \mathrm{kHz})$, and $t$ denotes time in seconds. The probe duration was $50 \mathrm{~ms}$, including 4-ms cosine squared rise/fall ramps. The probe level was $-20 \mathrm{~dB}$ full scale (FS), where $0 \mathrm{~dB}$ FS corresponds to a signal with peak amplitude at unity. To match the levels of the modulated and unmodulated probes, the level of the AM probe was adjusted after applying the amplitude modulation.

The probe was chosen to be short in duration $(50 \mathrm{~ms})$ to prevent (or minimize) noise adaptation from occurring in the early condition, and the modulation rate $(40 \mathrm{~Hz})$ was chosen so that the carrier frequency and the two sidebands (1460 and $1540 \mathrm{~Hz}$ ) were not perceived as separate frequency components. The spectrum of an AM sinusoid contains the carrier frequency $\left(f_{c}\right)$ and two sideband frequencies $\left(f_{c} \pm f_{m}\right)$. Because the bandwidth of every frequency channel in the sound processor was always greater than the $80 \mathrm{~Hz}$ separation between the two sidebands, we assumed that CI users

Table 1

CI user information. For bilateral CI users, data refer to the test ear. R: right; L: left; M: male; F: female; pps: pulses per second.

\begin{tabular}{|c|c|c|c|c|c|c|c|c|}
\hline Participant ID & Bilateral/unilateral & Better (test) ear & Gender & Etiology & Age (years) & CI use (months) & No. of channels used for testing & Pulse rate \\
\hline SA007 & Unilateral & $\mathrm{R}$ & M & Unknown & 49 & 126 & 11 & 1617 \\
\hline SA006 & Unilateral & $\mathrm{R}$ & $\mathrm{F}$ & Unknown & 48 & 126 & 11 & 1653 \\
\hline SA008 & Bilateral & $\mathrm{R}$ & M & Unknown & 16 & 129 & 10 & 1020 \\
\hline SA009 & Bilateral & $\mathrm{R}$ & M & Hereditary & 15 & 148 & 10 & 1538 \\
\hline SA010 & Bilateral & $\mathrm{R}$ & M & Unknown & 16 & 172 & 10 & 1099 \\
\hline SA011 & Bilateral & $\mathrm{L}$ & $\mathrm{F}$ & Unknown & 44 & 22 & 10 & 1754 \\
\hline SA013 & Bilateral & $\mathrm{R}$ & M & Hereditary & 8 & 83 & 12 & 1515 \\
\hline SA014 & Bilateral & $\mathrm{L}$ & M & Meningitis & 48 & 175 & 9 & 1846 \\
\hline SA015 & Bilateral & $\mathrm{L}$ & $\mathrm{F}$ & Meningitis & 35 & 147 & 11 & 1405 \\
\hline SA016 & Bilateral & $\mathrm{L}$ & $\mathrm{F}$ & Unknown & 74 & 150 & 10 & 1493 \\
\hline
\end{tabular}


could not detect the sidebands as separate frequency components, and that their responses during the task were based only on detecting the amplitude fluctuations in the carrier envelope. Fig. 1 shows that for a 10-channel processor, these fluctuations were largest for channels \#5 and \#6.

For bilateral CI users, AM detection thresholds were measured in quiet and in the presence of BBN $(0.1-10 \mathrm{kHz})$. The overall level of the $\mathrm{BBN}$ was fixed at $-30 \mathrm{~dB}$ FS (i.e., $10 \mathrm{~dB}$ lower than the probe level), thus its spectrum level was about $-70 \mathrm{~dB}$ FS (i.e., approximately $50 \mathrm{~dB}$ lower than the probe level). The BBN had a duration of $400 \mathrm{~ms}$ in all conditions and was gated with 4-ms onset and offset cosine squared ramps. The BBN was presented to the same ear as the probe (ipsilateral noise), to the ear opposite from the probe (contralateral noise), and diotically to the two ears (bilateral noise). For each of the three noise lateralities, AM detection thresholds were measured for AM carriers presented monaurally at the onset ('early' condition) and delayed $300 \mathrm{~ms}$ from the onset ('late' condition) of the noise. This amounted to 7 AM detection thresholds per listener: one in quiet plus six in noise ( 3 noise lateralities $\times 2$ temporal positions). For unilateral CI users, AM detection thresholds were measured in quiet and with ipsilateral noise only, and the hearing aid was turned off or removed during the measurements.

\subsection{Procedure}

AM detection thresholds were measured using a threealternative forced-choice ( $3 A F C$ ) adaptive procedure. Three intervals were presented to the participants accompanied by brief lights in a computer monitor, each of which lasted $400 \mathrm{~ms}$ (regardless of whether AM detections thresholds were measured in quiet or in noise, see below). Unmodulated carriers were presented in two of the three intervals and an amplitude modulated carrier (the 'target') was presented in another interval. The interval containing the target was chosen at random in each trial, and participants were instructed to identify the target interval by pressing a key on the computer keyboard. Feedback was given to the participants on the correctness of their responses. In the conditions with noise, the noise was presented in the three intervals and the lights turned on and off according to the onset and offset of the noise. In the quiet condition, the carrier started at the same time as the light or $300 \mathrm{~ms}$ after the light onset. ${ }^{2}$ The silence period between the intervals was $500 \mathrm{~ms}$ in duration and it was defined as the period between the offset and the onset of the brief lights.

The modulation depth of the AM probe $(m)$ decreased after two successive correct responses and increased after an incorrect response (two-down, one-up adaptive rule). AM threshold was thus defined as the modulation depth giving 70.7\% correct responses in the psychometric function (Levitt, 1971). The modulation depth varied using a logarithmic procedure as $20 \cdot \log _{10}(m)$, and $\mathrm{AM}$ detection threshold in $\mathrm{dB}$ was obtained. $m$ was equal to $0(-\infty \mathrm{dB})$ for unmodulated stimuli, and it was equal to $1(0 \mathrm{~dB})$ for $100 \%$ modulation. The initial modulation depth was $0 \mathrm{~dB}$ and changed by $4 \mathrm{~dB}$ until the second reversal in AM depth occurred and by $2 \mathrm{~dB}$ thereafter. The procedure continued until 12 reversals in modulation depth were obtained, and the AM detection threshold was calculated as the mean of the modulation depths at the last eight reversals. At least 3 AM thresholds were obtained for

\footnotetext{
2 Measuring AM detection thresholds for AM probes presented early and late after the light onset was not necessary but was technically convenient because the same software could be used to measure AM detection thresholds in noise and in quiet by adjusting only the noise level (i.e., in quiet the noise level was set to $-3000 \mathrm{~dB}$ FS). AM detection thresholds in quiet were not different when the AM probe started at the same time as the light onset or $300 \mathrm{~ms}$ after the light onset ( $p=0.346 ; N=6$; two-tailed, paired Student $t$-test).
}

each condition and their mean was taken as the AM threshold, except for participant SA007 in the ipsilateral-early condition, for whom 2 AM thresholds were measured. An AM detection threshold was discarded when the standard deviation within the measure was greater than $4 \mathrm{~dB}$. The proportion of discarded thresholds ranged from $0 \%$ to $25 \%$, depending on the participant, with a mean value across participants of $5 \%$.

The AM probe had only two modulation cycles. Therefore, the AM-detection task was hard and some participants found it difficult to discriminate the modulated from the unmodulated tones. Before testing, training was provided. Training always included measuring an AM detection threshold in ipsilateral noise at $-35 \mathrm{~dB}$ FS but if necessary the noise level, the modulation rate, and/or any other stimulus parameter was modified until the participant became able to reliably discriminate an AM probe with the characteristics specified in the study at $100 \%$ modulation depth. Despite training, some participants could not discriminate the AM probe in the early condition (see below).

AM detection thresholds in quiet were usually measured first, followed by the ipsilateral noise condition. Then, AM detection thresholds were measured with contralateral or bilateral noise. For each participant and noise laterality, the three (or more) AM detection thresholds in the early and late conditions were measured in random order. Because thresholds in quiet were the first to be measured and thus were more likely to be affected by insufficient training, an additional AM detection threshold in quiet was measured near the end of the experiment for five participants. This additional threshold was averaged with the previous measures to minimize possible training effects. For these five participants, the thresholds in quiet measured at the end of the experiment were only $1.4 \mathrm{~dB}$ better (on average) than those measured at the beginning of the experiment. In addition, when AM thresholds for a given participant and test condition tended to improve during the course of testing, additional AM detection threshold(s) were measured and averaged with the previous ones. Additional thresholds were measured for the $34 \%$ of the test conditions (calculated combining conditions in quiet and in noise across all participants).

\subsection{Apparatus}

A MATLAB software environment was used to perform signal processing and implement test procedures. Stimuli were generated digitally (at $20 \mathrm{kHz}$ sampling rate, 16-bit quantization), processed through the coding strategy, and the resulting electrical stimulation patterns delivered using the Research Interface Box 2 (RIB2; Department of Ion Physics and Applied Physics at the University of Innsbruck, Innsbruck, Austria) and each patient's implanted receiver/stimulator.

\subsection{Statistical analyses}

Repeated-measures analyses of the variance (RMANOVA) or paired Student's $t$-test were used to test for the statistical significance of temporal position and noise laterality on AM detection thresholds. Based on the results for NH listeners (Marrufo-Pérez et al., 2018b), we hypothesized that AM detection thresholds would be better (lower) in the late than in the early condition. Accordingly, we applied one-tailed tests when testing for differences in temporal position and two-tailed tests for all other comparisons. Independent-sample Student's $t$ tests or mixed analyses of the variance (ANOVA) were used to test for differences in AM detection threshold or in the magnitude of the temporal effect between $\mathrm{CI}$ users and NH listeners. The use of a mixed ANOVA is appropriate when the same measure is performed in two groups that belong to different populations (e.g., Detry and Ma, 2016). The 
comparison between $\mathrm{NH}$ and $\mathrm{CI}$ listeners seems reasonable because, as described below, the stimuli and conditions were identical across the two groups of participants, with the possible exception of a few decibels in stimulus levels. The results of the comparison, however, must be interpreted cautiously because the signal processing evoking an auditory nerve response is different for $\mathrm{NH}$ and $\mathrm{CI}$ listeners, and this can influence AM detection to some uncertain extent. For tests involving multiple groups or variables, post hoc pairwise comparisons were conducted using Bonferroni corrections for multiple comparisons. An effect was regarded as statistically significant when the null hypotheses could be rejected with $95 \%$ confidence $(p \leq 0.05)$.

\section{Results}

Individual AM detection thresholds are shown in Fig. 2. For SA007 and SA006, thresholds were measured only with ipsilateral noise because those participants were unilateral CI users. Three bilateral participants could not discriminate the AM probe in noise from the unmodulated tones in the early conditions, even after extensive training: SA009 in ipsi-, contra- and bilateral noise; SA010 in bilateral noise; and SA016 in ipsi- and bilateral noise. For these three participants, the AM thresholds in the early condition were arbitrarily set to $-1 \mathrm{~dB}$ (i.e., half the final step size in modulation depth), which was deemed more conservative than setting it to $0 \mathrm{~dB}$ for revealing any temporal effect on AM detection threshold. SA014 could not discriminate the AM probe presented in ipsilateral or bilateral noise, either in the early or the late conditions. This explains the missing data for SA014 in Fig. 2. Participants who could and could not discriminate the AM probe from the unmodulated tones were not different in age, time of $\mathrm{CI}$ use, number of channels or pulse rate (compare SA009, SA010, SA016 and SA014 with other

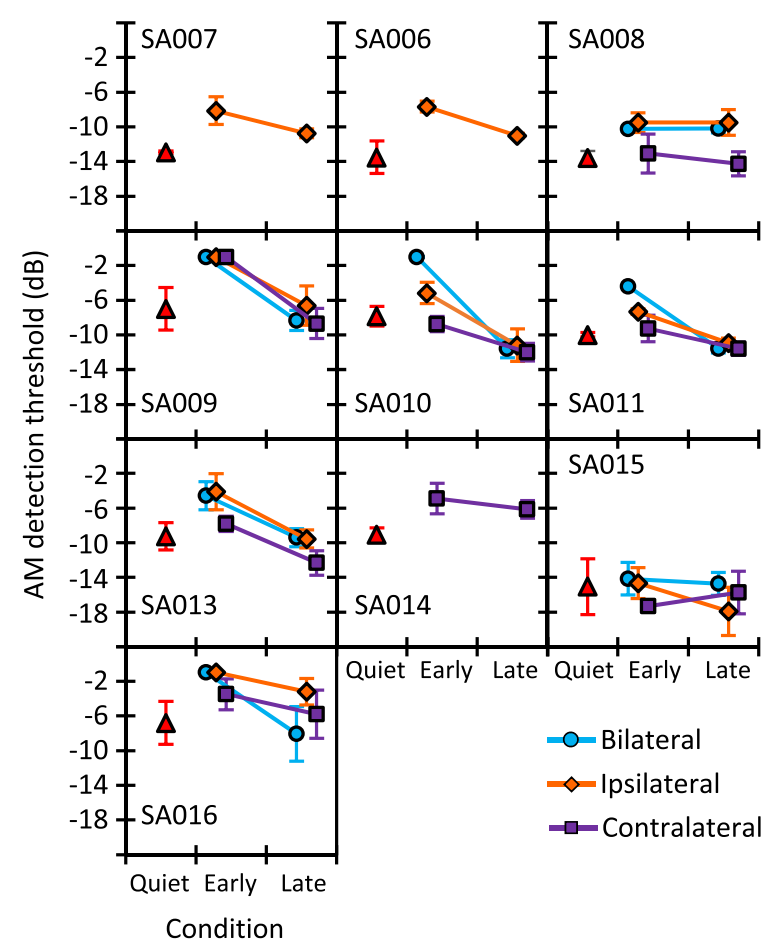

Fig. 2. Individual AM detection thresholds for $\mathrm{CI}$ users in quiet and in bilateral, ipsilateral and contralateral BBN. Each point is the mean of three or more measurements. AM thresholds in noise are represented for the early (probe and noise started at the same time) and late (probe started $300 \mathrm{~ms}$ after the noise onset) conditions. Error bars illustrate one standard deviation. participants in Table 1). In addition, they were not different in electrical dynamic range (not shown). Therefore, it remains uncertain why the participants in question could not discriminate AM from unmodulated tones.

Most individual participants showed a temporal effect or noise adaptation; i.e., better AM thresholds for the late than for the early conditions (Fig. 2). The group mean thresholds are shown by circles in Fig. $3 \mathrm{~A}$ and were calculated based on the data for the seven bilateral participants who could perform the task in the late condition (data for SA007, SA006 and SA014 were excluded). A twoway RMANOVA was conducted to test for the effects of temporal position (early versus late), noise laterality (bilateral, ipsilateral, or contralateral) and their interaction on AM detection thresholds. The analysis revealed a significant effect of temporal position $[F(1,6)=16.57, \quad p=0.004]$ and noise laterality $[F(2,12)=9.61$, $p=0.003]$. The interaction between temporal position and noise laterality was not significant $[F(2,12)=2.90, p=0.093]$, which indicates that the magnitude of the temporal effect was not statistically different across the three noise lateralities. Post hoc analyses revealed a significant temporal effect for the bilateral $($ mean $=5.4 \mathrm{~dB} ; p=0.005)$, ipsilateral $($ mean $=3.7 \mathrm{~dB} ; p=0.002)$ and contralateral (mean $=2.8 \mathrm{~dB} ; p=0.021$ ) noises (Fig. 3B).

Fig. 3 also shows AM thresholds and temporal effects for $\mathrm{NH}$ listeners ( $N=7$, data replotted from Marrufo-Pérez et al., 2018b). A comparison between AM detection thresholds for the present $\mathrm{CI}$ users and for $\mathrm{NH}$ listeners is warranted since the stimuli and conditions were similar across the two studies, including the probe level ( $70 \mathrm{~dB}$ SPL for NH versus $-20 \mathrm{~dB}$ FS for CI users, which corresponds to approximately $65 \mathrm{~dB}$ SPL in the clinical $\mathrm{CI}$ audio processors of MED-EL) and the noise level (60 dB SPL for $\mathrm{NH}$ versus $-30 \mathrm{~dB}$ FS for $\mathrm{CI}$ users, which corresponds to approximately
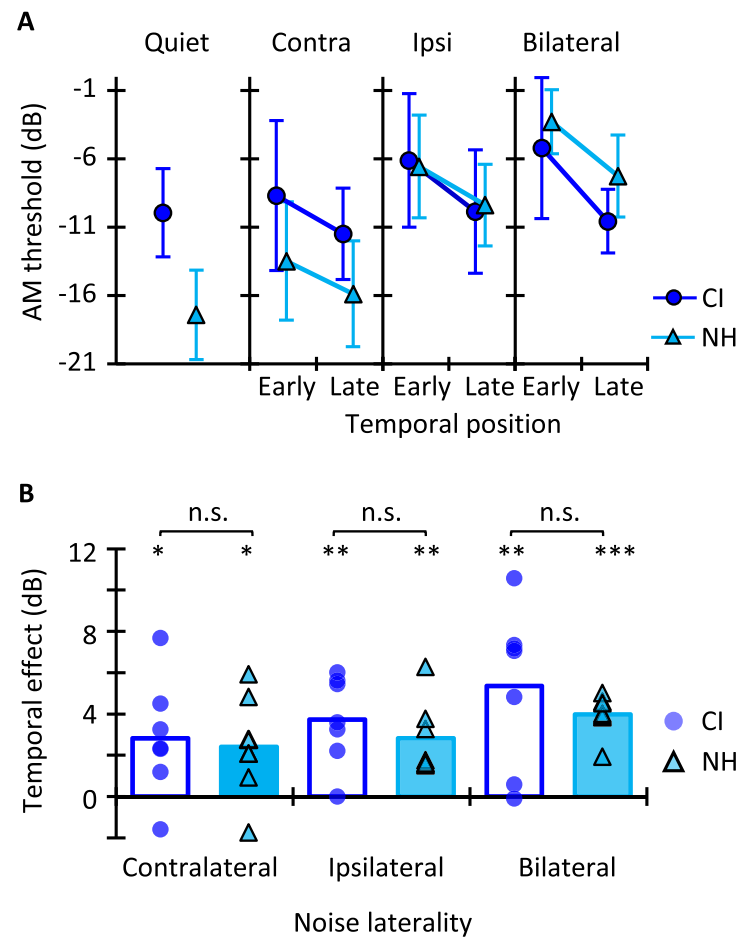

Fig. 3. A. Mean AM detection thresholds for $\mathrm{CI}$ users $(N=7)$ and $\mathrm{NH}$ listener $(N=7)$ in quiet and for contralateral, ipsilateral and bilateral noise in the early and late conditions. Data for NH listeners are re-plotted from Marrufo-Pérez et al. (2018b) and they are for the carrier level of $70 \mathrm{~dB}$ SPL and BBN level of $60 \mathrm{~dB}$ SPL. Error bars illustrate one standard deviation. B. Mean temporal effect magnitude (early minus late AM thresholds) for the three noise lateralities and for each group of participants. Asterisks indicate statistically significant differences: ${ }^{*} p \leq 0.05 ;{ }^{* *} p \leq 0.01 ;{ }^{* * *} p \leq 0.001$. 
$55 \mathrm{~dB}$ SPL). Fig. 3A shows that AM thresholds were different in most conditions for the two groups of participants. In quiet, AM thresholds were $7.5 \mathrm{~dB}$ better for $\mathrm{NH}$ listeners than for $\mathrm{CI}$ users $[t(12)=4.30, p<0.001$; independent samples $t$-test $]$. In noise, a mixed ANOVA with participant group (NH listeners versus $\mathrm{CI}$ users) as between-subjects factor and noise laterality and temporal position as within-subject factors revealed a significant interaction between group and noise laterality on AM thresholds $[F(2,24)=20.89 ; p<0.001]$. Post hoc pairwise comparisons with Bonferroni corrections, however, revealed no statistically significant differences in AM thresholds between $\mathrm{CI}$ users and $\mathrm{NH}$ listeners for bilateral $(p=0.142)$, ipsilateral $(p=0.995)$ or contralateral $(p=0.059)$ noise.

Finally, a mixed ANOVA with participant group as betweensubjects factor and noise laterality as within-subject factor was used to test for differences on the magnitude of the temporal effects between $\mathrm{NH}$ listeners and $\mathrm{CI}$ users. The analysis revealed no interaction between noise laterality and subject group $[F(2,24)=0.22 ; p=0.803]$. This indicates that the magnitude of the temporal effect was not statistically different for the two subject groups for any noise laterality (Fig. 3B).

\section{Discussion}

CI users tested with an experimental processing strategy without dynamic processing showed adaptation to noise in AM detection; that is, they showed better AM detection thresholds for an $A M$ probe presented in noise when the AM probe was delayed $300 \mathrm{~ms}$ from the noise onset than when the AM probe and the noise started at the same time. The magnitude of adaptation was not statistically different from that reported by Marrufo-Pérez et al. (2018b) for NH listeners tested using similar stimuli and experimental conditions (Fig. 3B). In addition, as for NH listeners, the magnitude of noise adaptation for CI users was not statistically different for ipsilateral, contralateral, and bilateral noise, although it tended to be slightly greater for bilateral noise (Fig. 3B).

Because the electrical stimulation delivered by CIs is independent from both the middle ear muscle reflex (MEMR) and the MOCR, the present results show that noise adaptation can occur without the MEMR or the MOCR. Furthermore, because the magnitude of noise adaptation was comparable for $\mathrm{NH}$ listeners and CI users, this shows that adaptation can be similar in magnitude with and without the MEMR or the MOCR, at least for the experimental conditions employed here. In addition, the existence of adaptation for contralateral noise suggests that for CI users at least, adaptation is not peripheral in origin and that it probably occurs beyond the auditory nerve, possibly in neurons that integrate stimuli from both ears. It would be wrong to conclude, however, that the MEMR and/or the MOCR do not play a role in noise adaptation for $\mathrm{NH}$ listeners, as discussed below.

\subsection{Comparison with other studies for $\mathrm{NH}$ listeners}

The comparison of the present results with studies for NH listeners other than that of Marrufo-Pérez et al. (2018b) is not straightforward because different studies have used different experimental designs and/or stimuli. For example, Almishaal et al. (2017) measured AM detection thresholds in quiet using a short (50 ms) monaural narrow-band noise carrier centered at $5 \mathrm{kHz}$ with a modulation rate of $20 \mathrm{~Hz}$. The carrier was presented in the presence and in the absence of an ipsilateral notched noise precursor of $200 \mathrm{~ms}$ in duration and $40 \mathrm{~dB}$ SPL. For a 65-dB SPL carrier, AM detection thresholds were $5 \mathrm{~dB}$ better with than without the precursor, an improvement slightly greater than the temporal effect of $3.7 \mathrm{~dB}$ reported here for $\mathrm{CI}$ users in ipsilateral noise (the most similar condition). Jennings et al. (2018) presented a short (50 ms) $2-\mathrm{kHz}$ carrier in quiet, modulated at $40 \mathrm{~Hz}$, at different levels with and without a notched noise precursor of $200 \mathrm{~ms}$ in duration and $40 \mathrm{~dB}$ SPL. For a 65-dB SPL carrier, NH listeners showed AM detection thresholds that were about $2 \mathrm{~dB}$ better with than without the precursor, a value smaller than the $3.7 \mathrm{~dB}$ reported here.

The results of Almishaal et al. (2017) and Jennings et al. (2018) were consistent with an activation of the MOCR by the precursor enhancing AM depth in the cochlear mechanical response. While the present findings show that noise adaptation can occur without the MOCR (or the MEMR), the present evidence is insufficient to reject the MOCR explanation for the data of Almishaal et al. (2017) or Jennings et al. (2018). In those studies, AM detection thresholds were measured for AM probes in quiet and preceded by an ipsilateral noise precursor while here, the AM probe was presented simultaneously with noise. Perhaps the activation of the MOCR by the precursor is responsible for the temporal enhancement of AM depth when the AM probe is presented in quiet but mechanisms different from the MOCR can enhance AM depth when the AM probe is presented in noise (see below). This difference in methodology might also explain why Jennings et al. (2018) found small or no temporal improvements in AM thresholds for HI listeners while we have found close-to-normal improvements for $\mathrm{CI}$ users even though neither $\mathrm{CI}$ users nor $\mathrm{HI}$ listeners are expected to have normal MOCR effects [the evidence for abnormal MOCR effects in $\mathrm{HI}$ listeners, however, is scarce and indirect; for existing evidence, see Frisina et al. (2007) and Zhu et al. (2007)].

\subsection{Possible mechanisms underlying adaptation to noise}

The present data demonstrate that the sensitivity to AM in noise can improve over time for listeners without a functional MOCR or MEMR. Although the physiological mechanism underlying this improvement remains uncertain, previous studies have revealed that adaptation processes not related with the MOCR can enhance the representation of AM in neural responses. For example, Coombs and Fay (1985) demonstrated that the spikes of goldfish saccular fibers were more synchronized to the amplitude modulations in the stimulus when neurons showed short-term adaptation (i.e., a progressive decrease in firing rate over the first $100 \mathrm{~ms}$ after the onset of the AM) than when they did not. They further showed that the synchronization to the amplitude modulations was even greater under conditions inducing long-term adaptation (firingrate adaptation with a time course of seconds or minutes). Neurons in the brainstem of frogs (Bibikov, 2002, 2013; Bibikov and Nizamov, 1996) or in the auditory nerve of cats stimulated with CIs (Hu et al., 2010) also increase their synchronization to AM progressively along time, i.e., synchronization increases as a function of firing rate adaptation. It is uncertain how firing rate adaptation can enhance AM coding but recent studies have revealed that firing rate adaptation is associated with dynamic-range adaptation. As firing rate adapts, the rate-level function of auditory neurons also adapts toward the most frequently occurring level in the sound (Fig. 3 in Wen et al., 2009). In other words, neurons show a displacement of the rate-level function (together with a decrement in firing rate) to adapt their dynamic range of operation to the sound-level distribution. This adaptation occurs in the auditory nerve (Wen et al., 2009, 2012) and at higher levels in the auditory pathway (Dean et al., 2005, 2008; Watkins and Barbour, 2008), and can last from hundreds of milliseconds to seconds (Dean et al., 2008). Here, the probe level at the probe channels (\#5 and \#6) (Fig. 1) were within $10 \mathrm{~dB}$ of the noise levels (Fig. 4). Therefore, the rate-level functions of auditory neurons in the late condition could have shifted horizontally toward the noise level (the longest stimulus), and thus facilitated AM coding when the AM fell within 

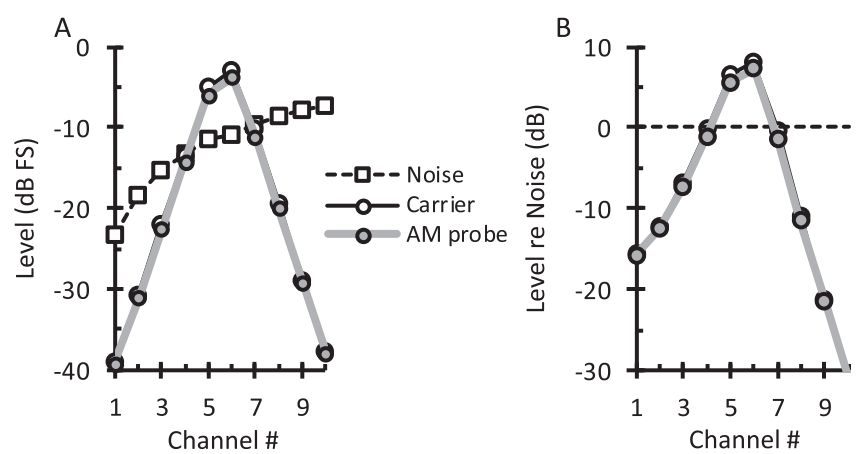

Fig. 4. A. Root-mean-square levels (in $\mathrm{dB} F S$ ) at the output of a 10-channel processor for the present stimuli. Results are shown for the noise $(-30 \mathrm{~dB}$ FS $)$ and for unmodulated $(\mathrm{m}=0)$ and $100 \%$ modulated $(\mathrm{m}=1)$ carrier tones $(1.5-\mathrm{kHz},-20 \mathrm{~dB} \mathrm{FS})($ see inset). Note that the output level to the unmodulated carrier and modulated carriers is largest for Channels \#5 and \#6. The output level for the noise increases with increasing channel number because the noise was broadband $(0.1-10 \mathrm{kHz})$ and the bandwidth of the filters in the $\mathrm{CI}$ processor increased with increasing channel number (see Methods). B. Carrier-to-noise ratio (dB) for each processor channel.

the adapted rate-level curve (as depicted in Fig. 1C of MarrufoPérez et al., 2018b).

The detection threshold for pure tones embedded in noise are typically higher for tones presented at the noise onset than for tones delayed from the noise onset, a phenomenon known as transient masking or 'overshoot' (Bacon and Moore, 1987). If the AM carrier tone were undetectable in the early condition, or were less audible in the early than in the late condition, this may have caused AM detection thresholds to be worse in the early condition. It is unlikely, however, that this explains the temporal improvement in AM detection over time. First, all CI users reported to hear a tone in the early condition even when they were not able to discriminate the modulated from the unmodulated tones. Second, $\mathrm{NH}$ listeners show temporal improvements in AM-in-noise sensitivity even when the carriers are presented at equal sensation levels in the early as in the late conditions (Marrufo-Pérez et al., 2018b).

AM detection thresholds are known to be worse when the AM probe is presented with a simultaneous masker modulated at the same rate as the probe, even when the masker carrier is remote in frequency from the probe carrier (Yost et al., 1989). This phenomenon is referred to as modulation detection interference (MDI) because it is thought to reflect a difficulty in segregating the masker AM from the probe AM (Hall and Grose, 1991; Yost et al., 1989; Yost and Sheft, 1990). One might think that to perform the present task, listeners had to segregate the AM of the probe from the inherent envelope fluctuations of the noise. Indeed, some aspects in the present data appear consistent with MDI. For example, MDI occurs for ipsilateral and contralateral maskers (Bacon and Opie, 1994; Yost and Sheft, 1990); MDI occurs for cochlear implant users (Richardson et al., 1998); and more importantly, MDI decreases as the AM probe is delayed from the onset of the masker carrier (e.g., Hall and Grose, 1991; Oxenham and Dau, 2001). Therefore, perhaps the temporal improvement in AM-in-noise sensitivity reported here and elsewhere may be reflecting a decrease of MDI over time. To our knowledge, the physiological mechanisms for MDI or for the decrease of MDI over time are uncertain, but some perceptual characteristics of MDI appear inconsistent with dynamic range adaptation. For example, MDI occurs even for AM masker carriers as remote as two octaves below or above the AM probe (Yost et al. 1989), and dynamic range adaptation presumably occurs only when the frequency spectrum of the adapting sounds is within the frequency response area of the adapted neuron. Therefore, if the temporal improvements in AM-in-noise sensitivity reported here and elsewhere (Marrufo-Pérez et al., 2018b) were a manifestation of MDI, then dynamic range adaptation may be insufficient to explain them; at least dynamic range adaptation in the auditory nerve.

However, not every aspect of the present data is consistent with MDI. AM detection thresholds in quiet were not statistically different from those in simultaneous contralateral noise in the early condition (two-tailed paired Student's t-test, $N=7, t=1.22$, $p=0.267$ ), indicating that MDI did not occur for contralateral noise. Yet AM detection thresholds in contralateral noise were significantly better in the late than in the early condition (one-tailed paired Student's $t$-test, $N=7, t=2.60, p=0.021$ ). In other words, AM sensitivity in contralateral noise improved over time in the absence of statistically significant MDI. In addition, MDI is largest when the modulation rate of the masker matches the modulation rate of the probe (Moore et al., 1995; Yost et al., 1989). Here, for a $100 \%$ modulated carrier in ipsilateral noise, the $40-\mathrm{Hz}$ component in the envelope spectra (the most interfering frequency) was at least $10 \mathrm{~dB}$ lower for channels remote from the probe channels than for the probe channels (\#5 and \#6) (Fig. 5). This undermines the idea that the $40-\mathrm{Hz}$ envelope fluctuations in the 'noise' channels (i.e. channels remote from the probe channels) strongly interfered with the probe AM. We note, however, that components in the noise envelope spectrum different from the $40-\mathrm{Hz}$ component may also be contributing to MDI and affect AM detection thresholds to some uncertain extent (Ewert and Dau, 2000).

In any case, whether or not the present temporal improvements in AM sensitivity reflect a decrease of MDI over time, the present study shows that those improvements can occur without the MOCR or the MEMR.

\subsection{AM thresholds for $\mathrm{NH}$ listeners and $\mathrm{CI}$ users}

The level of the AM probe used here to test $\mathrm{CI}$ users $(-20 \mathrm{~dB}$ FS, or about $65 \mathrm{~dB}$ SPL) was close to that used by Marrufo-Perez et al. (2018b) to test $\mathrm{NH}$ listeners (70 dB SPL), and yet AM detection thresholds in quiet were better for $\mathrm{NH}$ listeners than for $\mathrm{CI}$ users (Fig. 3A). This result is consistent with the results from Park et al. (2015) and Jin et al. (2014), who presented the AM probe at the same level for the two participant groups and found worse performance for $\mathrm{CI}$ users than for $\mathrm{NH}$ listeners. The present result, however, differs from the results of other studies that reported similar (Donaldson and Viemeister, 2000; Shannon, 1992) or even better (Shannon, 1992) AM detection thresholds for CI users than for $\mathrm{NH}$ listeners. The reason for this difference is uncertain but AM detection thresholds for $\mathrm{CI}$ users vary depending on multiple factors. For example, whereas AM sensitivity is independent of carrier level for $\mathrm{NH}$ listeners (except at very low listening levels;

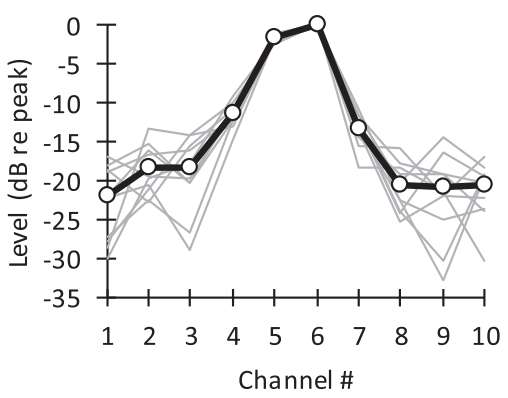

Fig. 5. Level of the $40-\mathrm{Hz}$ envelope component at the output of a 10 -channel CI processor for an AM probe $(m=1)$ in noise. The level is expressed in $d B$ relative to the peak value across channels. To illustrate the effect of noise variability, thin lines illustrate values for 10 different noises. The thick line with open circles illustrates mean values across the 10 noise instances. 
Viemeister, 1979), AM sensitivity for CI users improves markedly as carrier level increases (typically expressed as percent dynamic range; Fu, 2002; Galvin and Fu, 2005; Fraser and McKay, 2012; Pfingst et al., 2008; Donaldson and Viemeister, 2000), which can result in AM sensitivity to be even better for $\mathrm{CI}$ users than for $\mathrm{NH}$ listeners at high carrier levels (Shannon, 1992). In addition, AM thresholds for $\mathrm{CI}$ users can vary depending on electrode location (Pfingst et al., 2008) or stimulation rate (Galvin and Fu, 2005; Fraser and McKay, 2012).

For both NH listeners and CI users, AM detection thresholds were significantly lower (better) in quiet than in ipsilateral or bilateral noise in the early condition, where adaptation has not yet occurred $(p<0.05$, Fig. 3A). However, adding noise increased (worsened) AM thresholds comparatively more for $\mathrm{NH}$ listeners than for CI users. For example, thresholds worsened $81 \%$ for $\mathrm{NH}$ listeners versus $48 \%$ for CI users from quiet to the bilateral noiseearly condition, suggesting that $\mathrm{CI}$ users were relatively less affected by noise than $\mathrm{NH}$ listeners. The reason is uncertain but may be related to stochastic resonance. For CI users, AM sensitivity improves when low-level noise is added to the signal (Chatterjee and Robert, 2001; Chatterjee and Oba, 2005). The interpretation is that low-level noise possibly restores the stochastic responses observed in the auditory nerve during acoustic listening but not during electrical stimulation, and this restoration would improve auditory perception. Perhaps, the comparatively smaller masking effect of noise for CI users than for the $\mathrm{NH}$ listeners in the early condition is due to $\mathrm{CI}$ users lacking the benefits of natural stochastic resonance in quiet.

\subsection{Open issues}

While the present findings suggest that noise adaptation can occur without the MOCR (or the MEMR), we cannot rule out that the MOCR (and/or the MEMR) contributes to some uncertain extent to noise adaptation for $\mathrm{NH}$ listeners. $\mathrm{CI}$ users and $\mathrm{NH}$ listeners may have similar noise adaptation (Fig. 3B) because the MOCR is not involved in adaptation for $\mathrm{NH}$ listeners or because $\mathrm{CI}$ users are benefiting from different and/or enhanced neural adaptation mechanisms. For example, it is conceivable that neural plasticity might enhance neural dynamic range adaptation for CI users compared to $\mathrm{NH}$ listeners to compensate somehow for the lack of an MOCR.

Some issues remain open. For example, we have assumed that because auditory nerve fibers stimulated with electric pulse trains show firing rate adaptation (Zhang et al., 2007; Hu et al., 2010) and short-term firing rate adaptation and dynamic range adaptation are likely mediated by a common neural mechanism (Wen et al., 2012), then dynamic range adaptation also occurs for electrical stimulation. This, however, is yet to be shown. Second, to discard the potential confound of transient masking, it would be useful to objectively corroborate (e.g., using envelope following responses) that for an AM probe in simultaneous noise, AM depth is gradually better represented in the neural response as the probe is delayed from the noise onset. Finally, throughout the present study, we have considered neural dynamic range adaptation as a potential mechanism underlying the temporal improvement in AM-in-noise detection. This, however, is only speculation and the actual mechanism is yet to be elucidated. As reasoned above, if the improvement were reflecting a decrease of MDI over time, then some aspects of MDI may not be consistent with dynamic range adaptation.

\subsection{Final remark}

Assessing the specific contribution of the MOCR to noise adaptation for $\mathrm{NH}$ listeners using psychoacoustical tasks might turn out impossible because the noise used to evoke the MOCR can also evoke/induce other adaptation mechanisms such as, for example, dynamic range adaptation. However, insofar as the present condition where the AM probe is embedded in noise is representative of ecological listening situations where speech is presented in competition with simultaneous noise, the present findings together with those of Marrufo-Perez et al. (2018a) for word-in-noise recognition demonstrate that, when MOCR effects are not available, mechanisms other than the MOCR can facilitate AM-in-noise detection and word-in-noise recognition.

\section{Conclusions}

1. Cochlear implant users show $\sim 4 \mathrm{~dB}$ better $\mathrm{AM}$ detection thresholds when the AM carrier is presented later rather than earlier in broadband noise.

2. The improvement occurs for bilateral, ipsilateral, and contralateral noises, and the magnitude is not statistically different across noise lateralities.

3. The magnitude of improvement is not statistically different from that for $\mathrm{NH}$ listeners when AM detection is measured using similar stimuli and experimental conditions.

4. Because the electrical stimulation delivered by the cochlear implant is independent from the middle-ear muscle reflex or the medial olivocochlear reflex, the present results demonstrate that mechanisms unrelated to these reflexes can enhance AM cues and facilitate the detection of AM in noise over time.

\section{Conflict of interest}

The authors declare no competing interests.

\section{Acknowledgements}

Work supported by a doctoral contract of the University of Salamanca and Banco Santander to M.I.M.-P., and by the European Regional Development Fund and the Spanish Ministry of Economy and Competitiveness (Grant BFU2015-65376-P) to E.A.L.-P. We thank MED-EL GmbH and Dr. Otto Peter from the University of Innsbruck for technical support and equipment.

\section{Appendix A. Supplementary data}

Supplementary data to this article can be found online at https://doi.org/10.1016/j.heares.2019.03.017.

\section{References}

Almishaal, A., Bidelman, G.M., Jennings, S.G., 2017. Notched-noise precursors improve detection of low-frequency amplitude modulation. J. Acoust. Soc. Am. $141,324-333$.

Backus, B.C., Guinan, J.J., 2006. Time-course of the human medial olivocochlear reflex. J. Acoust. Soc. Am. 119, 2889-2904.

Bacon, S.P., Moore, B.C.J., 1987. Transient masking and the temporal course of simultaneous tone-on-tone masking. J. Acoust. Soc. Am. 81, 1073-1077.

Bacon, S.P., Opie, J.M., 1994. Monotic and dichotic modulation detection interference in practised and unpractised subjects. J. Acoust. Soc. Am. 95, 2637-2641.

Ben-David, B.M., Tse, V.Y., Schneider, B.A., 2012. Does it take older adults longer than younger adults to perceptually segregate a speech target from a background masker? Hear. Res. 290, 55-63.

Ben-David, B.M., Avivi-Reich, M., Schneider, B.A., 2016. Does the degree of linguistic experience (native versus nonnative) modulate the degree to which listeners can benefit from a delay between the onset of the maskers and the onset of the target speech? Hear. Res. 341, 9-18.

Berlin, C.I., Hood, L.J., Hurley, A.E., Wen, H., Kemp, D.T., 1995. Binaural noise suppresses linear click-evoked otoacoustic emissions more than ipsilateral or contralateral noise. Hear. Res. 87, 96-103. 
Bibikov, N.G., 2002. Coding of amplitude-modulated signals in the cochlear nucleus of a grass frog. Acoust Phys. 48, 447-460.

Bibikov, N.G., 2013. Adaptation of differential sensitivity of auditory system neurons to amplitude modulation after abrupt change of signal level. J. Evol. Biochem. Physiol. 49, 66-77.

Bibikov, N.G., Nizamov, S.V., 1996. Temporal coding of low-frequency amplitude modulation in the semicircularis of the grass frog. Hear. Res. 101, 23-44.

Boyd, P.J., 2006. Effects of programming threshold and maplaw settings on acoustic thresholds and speech discrimination with the MED-EL COMBI 40+ cochlear implant. Ear Hear. 27, 608-618.

Cervera, T., Ainsworth, W.A., 2005. Effects of preceding noise on the perception of voiced plosives. Acta Acust United Acust 91, 132-144.

Cervera, T., Gonzalez-Alvarez, J., 2007. Temporal effects of preceding bandpass and band-stop noise on the recognition of voiced stops. Acta Acust United Acust 93, 1036-1045.

Chatterjee, M., Oba, S.I., 2005. Noise improves modulation detection by cochlear implant listeners at moderate carrier levels. J. Acoust. Soc. Am. 118, 993-1002.

Chatterjee, M., Robert, M.E., 2001. Noise enhances modulation sensitivity in cochlear implant listeners: stochastic resonance in a prosthetic sensory system? J Assoc Res Otolaryngol 2, 159-171.

Coombs, S., Fay, R.R., 1985. Adaptation effects on amplitude modulation detection: behavioral and neurophysiological assessment in the goldfish auditory system. Hear. Res. 19, 57-71.

Cooper, N.P., Guinan, J.J., 2003. Separate mechanical processes underlie fast and slow effects of medial olivocochlear efferent activity. J. Physiol. 548, 307-312.

Cooper, N.P., Guinan, J.J., 2006. Efferent-mediated control of basilar membrane motion. J. Physiol. 576, 49-54.

Dean, I., Harper, N.S., McAlpine, D., 2005. Neural population coding of sound leve adapts to stimulus statistics. Nat. Neurosci. 8, 1684-1689.

Dean, I., Robinson, B.L., Harper, N.S., McAlpine, D., 2008. Rapid neural adaptation to sound level statistics. J. Neurosci. 28, 6430-6438.

Detry, M.A., Ma, Y., 2016. Analyzing repeated measurements using mixed models J. Am. Med. Assoc. 315, 407-408.

Donaldson, G.S., Viemeister, N.F., 2000. Intensity discrimination and detection of amplitude modulation in electric hearing. J. Acoust. Soc. Am. 108, 760-763.

Ewert, S.D., Dau, T., 2000. Characterizing frequency selectivity for envelope fluctuations. J. Acoust. Soc. Am. 108, 1181-1196.

Fraser, M., McKay, C.M., 2012. Temporal modulation transfer functions in cochlear implantees using a method that limits overall loudness cues. Hear. Res. 283 56-69.

Frisina, R.D., Newman, S.R., Zhu, X., 2007. Auditory efferent activation in CBA mice exceeds that of C57s for varying levels of noise. J. Acoust. Soc. Am. 121, EL29-EL34.

Fu, Q.J., 2002. Temporal processing and speech recognition in cochlear implant users. Neuroreport 13, 1635-1639.

Galvin, J.J., Fu, Q.J., 2005. Effects of stimulation rate, mode and level on modulation detection by cochlear implant users. J Assoc Res Otolaryngol 6, 269-279.

Guinan, J.J., 1996. Efferent physiology. In: Dallos, P., Popper, A.N., Fay, R.R. (Eds.), The Cochlea. Springer Handbook of Auditory Research, vol. 8. Springer, New York, pp. $435-502$.

Guinan, J.J., 2006. Olivocochlear efferents: anatomy, physiology, function, and measurements of efferent effects in humans. Ear Hear. 27, 589-607.

Hall, J.W., Grose, J.H., 1991. Some effects of auditory grouping factors on modulation detection interference (MDI). J. Acoust. Soc. Am. 90, 3028-3035.

Hu, N., Miller, C.A., Abbas, P.J., Robinson, B.K., Woo, J., 2010. Changes in auditory nerve responses across the duration of sinusoidally amplitude-modulated electric pulse-train stimuli. J Assoc Res Otolaryngol 11, 641-656.

Jennings, S.G., Chen, J., Fultz, S.E., Ahlstrom, J.B., Dubno, J.R., 2018. Amplitude modulation detection with a short-duration carrier: effects of a precursor and hearing loss. J. Acoust. Soc. Am. 143, 2232-2243.

Jin, S.H., Liu, C., Sladen, D.P., 2014. The effects of aging on speech perception in noise: comparison between normal-hearing and cochlear-implant listeners. J. Am. Acad. Audiol. 25, 656-665.
Levitt, H., 1971. Transformed up-down methods in psychoacoustics. J. Acoust. Soc. Am. 49, 467-677.

Lopez-Poveda, E.A., 2018. Olivocochlear efferents in animals and humans: from anatomy to clinical relevance. Front. Neurol. 9, 197.

Lopez-Poveda, E.A., Eustaquio-Martin, A., Stohl, J.S., Wolford, R.D., Schatzer, R., Wilson, B.S., 2016. A binaural cochlear implant sound coding strategy inspired by the contralateral medial olivocochlear reflex. Ear Hear. 37, 138-148.

Maison, S., Micheyl, C., Andeol, G., Gallego, S., Collet, L., 2000. Activation of medial olivocochlear efferent system in humans: influence of stimulus bandwidth. Hear: Res. 140, 111-125.

Marrufo-Pérez, M.I., Eustaquio-Martín, A., Lopez-Poveda, E.A., 2018a. Adaptation to noise in human speech recognition unrelated to the medial olivocochlear reflex. J. Neurosci. 38, 4138-4145.

Marrufo-Pérez, M.I., Eustaquio-Martín, A., Bascuas, L.E., Lopez-Poveda, E.A., 2018b. Temporal effects on monaural amplitude-modulation sensitivity in ipsilateral, contralateral and bilateral noise. J Assoc Res Otolaryngol 19, 147-161.

Moore, B.C.J., Sek, A., Shailer, M.J., 1995. Modulation discrimination interference for narrow-band noise modulators. J. Acoust. Soc. Am. 97, 2493-2497.

Murugasu, E., Russell, I.J., 1996. The effect of efferent stimulation on basilar membrane displacement in the basal turn of the Guinea pig cochlea. J. Neurosci. 16, 325-332.

Oxenham, A.J., Dau, T., 2001. Modulation detection interference: effects of concurrent and sequential streaming. J. Acoust. Soc. Am. 110, 402-408.

Park, M.H., Won, J.H., Horn, D.L., Rubinstein, J.T., 2015. Acoustic temporal modulation detection in normal-hearing and cochlear implanted listeners: effects of hearing mechanism and development. J Assoc Res Otolaryngol 16, 389-399.

Pfingst, B.E., Burkholder-Juhasz, R.A., Xu, L., Thompson, C.S., 2008. Across-site patterns of modulation detection in listeners with cochlear implants. J. Acoust. Soc. Am. 123, 1054-1062.

Richardson, L.M., Busby, P.A., Clark, G.M., 1998. Modulation detection interference in cochlear implant subjects. J. Acoust. Soc. Am. 104, 442-452.

Shannon, R.V., 1992. Temporal modulation transfer functions in patients with cochlear implants. J. Acoust. Soc. Am. 91, 2156-2164.

Sheft, S., Yost, W.A., 1990. Temporal integration in amplitude modulation detection. J. Acoust. Soc. Am. 88, 796-805

Viemeister, N.F., 1979. Temporal modulation transfer functions based upon modulation thresholds. J. Acoust. Soc. Am. 66, 1364-1380.

Warr, W.B., Guinan, J.J., 1979. Efferent innervation in the organ of Corti: two separate systems. Brain Res. 173, 152-155.

Watkins, P.V., Barbour, D.L., 2008. Specialized neuronal adaptation for preserving input sensitivity. Nat. Neurosci. 11, 1259-1261.

Wen, B., Wang, G.I., Dean, I., Delgutte, B., 2009. Dynamic range adaptation to sound level statistics in the auditory nerve. J. Neurosci. 29, 13797-13808.

Wen, B., Wang, G.I., Dean, I., Delgutte, B., 2012. Time course of dynamic range adaptation in the auditory nerve. J. Neurophysiol. 108, 69-82.

Wilson, B.S., Finley, C.C., Lawson, D.T., et al., 1991. Better speech recognition with cochlear implants. Nature 352, 236-238.

Winslow, R.L., Sachs, M.B., 1988. Single-tone intensity discrimination based on auditory-nerve rate responses in backgrounds of quiet, noise, and with stimulation of the crossed olivocochlear bundle. Hear. Res. 35, 165-189.

Yost, W.A., Sheft, S., 1990. A comparison among three measures of cross-spectral processing of amplitude modulation with tonal signals. J. Acoust. Soc. Am. 87, 897-900.

Yost, W.A., Sheft, S., Opie, J., 1989. Modulation interference in detection and discrimination of amplitude modulation. J. Acoust. Soc. Am. 86, 2138-2147.

Zhang, F., Miller, C.A., Robinson, B.K., Abbas, P.J., Hu, N., 2007. Changes across time in spike rate and spike amplitude of auditory nerve fibers stimulated by electric pulse trains. J Assoc Res Otolaryngol 8, 356-372.

Zhu, X., Vasilyeva, O.N., Kim, S., Jacobson, M., Romney, J., Waterman, M.S., Tuttle, D., Frisina, R.D., 2007. Auditory efferent feedback system deficits precede agerelated hearing loss: contralateral suppression of otoacoustic emissions in mice. J. Comp. Neurol. 503, 593-604. 